\title{
HUMAN CORONAVIRUS 229E CAN USE CD209L (L-SIGN) TO ENTER CELLS
}

\author{
Scott A. Jeffers, Erin M. Hemmila, and Kathryn V. Holmes*
}

\section{INTRODUCTION}

The primary receptor for SARS-CoV is ACE2, a metallopeptidase expressed on membranes of renal and cardiovascular tissues as well as the gastrointestinal tract. ${ }^{1-3}$ Marzi et al., showed that retroviral pseudotypes bearing the SARS-CoV glycoprotein could also bind to DC-SIGN and CD209L (also called L-SIGN or DC-SIGNR) on cell membranes, but not use these C-type lectins to enter cells. ${ }^{4}$ We showed that CD209L, which is expressed on sinusoidal endothelial cells of the liver, endothelial cells of the lymph nodes, Peyer's patches, capillaries in the villous lamina propria of the terminal ileum, and in type II alveolar cells and endothelial cells in the lung, has receptor activity for SARS-CoV. ${ }^{5}$ Briefly, Chinese hamster ovary $(\mathrm{CHO})$ cells, which are refractory to binding of SARS-CoV spike glycoprotein and entry of SARS-CoV, were transduced with a human lung cDNA library in a retroviral vector. Cells that bound soluble SARS-CoV spike glycoprotein were detected by flow cytometry and inoculated with SARS-CoV under BSL-3 conditions. Virus entry and viral subgenomic RNA and protein synthesis were detected by RT-PCR and immunofluorescence. Less than $1 \%$ of the cells expressed SARS-CoV nucleocapsid protein 24 hours after inoculation. CD209L cDNA was cloned from subcloned cells positive for both binding of SARS-CoV spike and expression of SARS-CoV subgenomic RNA and N protein. Transfection of CHO cells with cDNA encoding CD209L made these cells as susceptible to SARS-CoV as the retrovirus transduced cells.

DC-SIGN on dendritic cells has been shown to act as an attachment factor for Ebola virus, HIV-1, and other enveloped viruses. ${ }^{6}$ The dendritic cells, which are professional antigen-presenting cells, can present virus to macrophages or $\mathrm{CD} 4^{+} \mathrm{T}$-cells that express the appropriate receptor (CD4 and co-receptors for HIV-1). Thus DC-SIGN is said to mediate infection in trans. ${ }^{\text {? }}$

Our data suggest that CD209L may act as an alternative weak receptor for SARSCoV. Coronaviruses bud from the ER-Golgi intermediate compartment (ERGIC) and are believed to be released from cells by exocytosis. ${ }^{8,9}$ The maturation of virus in the ERGIC

*University of Colorado Health Sciences Center at Fitzsimons, Aurora, Colorado. 
may limit the amount of trimming of $N$-linked glycans on spike by endoglycosidases in the Golgi. We postulate that glycans on the coronavirus S proteins may be predominantly composed of the high mannose type rather than the more complex glycans on the spikes of enveloped viruses that bud from the plasma membrane. SARS-CoV may bind to CD209L on the plasma membrane through high mannose glycans on S, which may then mediate relatively inefficient virus entry. We have begun to study the roles of C-type lectins in entry of other coronaviruses such as human coronavirus (HCoV)-229E in addition to SARS-CoV.

\section{RESULTS AND DISCUSSION}

HCoV-229E uses human aminopeptidase N (hAPN) as its principal receptor ${ }^{10}$ and can also use feline APN as an alternative receptor. ${ }^{11}$ To determine whether the human coronavirus, $\mathrm{HCoV}-229 \mathrm{E}$, could also use CD209L as a receptor, we used flow cytometry to compare binding of purified $\mathrm{HCoV}-229 \mathrm{E}$ virions to cells expressing hAPN (MRC5), CD209L (CHO/CD209L) or neither receptor protein (CHO) (Table 1). HCoV-229E virions bound equally well to MRC5 cells via hAPN and $\mathrm{CHO} / \mathrm{CD} 209 \mathrm{~L}$ cells via CD209L.

Virus entry and expression of viral proteins were compared in $\mathrm{CHO}$ cells, $\mathrm{CHO}$ cells expressing CD209L, and mouse cells expressing hAPN. In cells that did not express hAPN or CD209L no viral antigen was detected at any time. However, in cells that expressed hAPN or CD209L viral antigens were observed 8 hours after virus inoculation. Twenty-four and 48 hours after inoculation, the hAPN-expressing cells were all positive for viral antigen. In contrast, fewer CD209L-expressing cells expressed viral antigens at 24 hours; however, by 48 hours after inoculation CD209L-expressing CHO cells were all positive for viral antigens. The pattern of antigen staining in the CD209L cells was granular with patches of bright cytoplasmic fluorescence; whereas staining in hAPN expressing cells was more uniform and bright throughout the cytoplasm. Seventy-two hours after inoculation the hAPN-expressing cells had high levels of cytopathic effect (CPE, large areas of rounded cells), whereas, the CD209L-expressing CHO cells showed no $\mathrm{CPE}$ and had formed a confluent monolayer of antigen positive cells. Thus, HCoV$229 \mathrm{E}$ entered cells that expressed CD209L, viral antigens were detected by immunofluorescence assay up to 72 hours after inoculation, and virus infection spread from cellto-cell in the culture. These data suggest that CD209L can act as a weak receptor for entry of HCoV-229E, but has less HCoV-229E receptor activity than hAPN. No infectious

Table 1. Binding of HCoV-229E to cell lines.

\begin{tabular}{lc}
\hline \multicolumn{1}{c}{ Cells } & $\begin{array}{c}\text { \% Cells that bind } \\
\text { virus* }\end{array}$ \\
\hline CHO & $<5$ \\
MRC5 & 97 \\
CHO/CD209L & 96 \\
\hline$*$ Virus binding was detected by flow cytometry \\
with monoclonal antibody 511H6 directed against \\
the viral spike glycoprotein.
\end{tabular}


virus was released into the medium of CD209L-expressing CHO cells, as determined by plaque assay. These data suggest that CD209L allows entry of HCoV-229E into some cells leading to synthesis of viral RNA and proteins. Possibly, CD209L on cell membranes traps newly formed virus preventing release of virus into the medium, while still allowing cell-to-cell spread of infection.

In order to further examine the interaction of CD209L with $\mathrm{HCoV}-229 \mathrm{E}$ spike, soluble CD209L with a 6-His tag was expressed in bacteria and purified to near homogeneity using mannose affinity chromatography and nickel affinity chromatography. Cell surfaces are replete with high-mannose glycans. To determine if purified soluble CD209L could bind to high mannose glycans on cells, trypsinized cells that express CD209L, hAPN, or neither receptor were incubated with $200 \mu \mathrm{g}$ of soluble CD209L for 1 hour at $4^{\circ} \mathrm{C}$. FACS analysis with anti-CD209L antibody showed that soluble CD209L bound to $\mathrm{CHO}$ cells expressing CD209L, MRC5 cells expressing hAPN, and control $\mathrm{CHO}$ cells that expressed neither receptor (Table 2).

To examine the effects of soluble CD209L on binding of HCoV-229E to cell lines, various concentrations of the purified soluble CD209L protein were incubated with purified $\mathrm{HCoV}-229 \mathrm{E}$ virions for 1 hour at $4^{\circ} \mathrm{C}$. The virions were then incubated with trypsinized CHO cells expressing CD209L, MRC5 cells expressing hAPN, or control $\mathrm{CHO}$ cells expressing neither receptor. Virus binding was detected by flow cytometry with monoclonal anti-229E spike antibody $511 \mathrm{H} 6$. Table 3 shows that lower levels of soluble CD209L $(50 \mu \mathrm{g} / \mathrm{ml})$ significantly blocked binding of HCoV-229E virions to hAPN or CD209L in cell membranes. However, larger amounts of soluble CD209L $(200 \mu \mathrm{g} / \mathrm{ml})$ increased binding of virions to CD209L and hAPN-expressing cells. Interestingly, virions incubated with $200 \mu \mathrm{g} / \mathrm{ml}$ of soluble CD209L bound to CHO cells that do not express either hAPN or CD209L. The soluble CD209L protein apparently acted as a bridge between the virions and the cellular surface. It is possible that binding of CD209L treated virions to $\mathrm{CHO}$ cells occurred when the carbohydrate recognition domain of soluble tetrameric CD209L bound to glycans on the surface of the virion, to glycans on soluble tetrameric CD209L and to glycans on the cell surface. Perhaps other soluble C-type lectins in the lung, such as surfactant protein D (SP-D), could also act as a bridge to bind virions to cells. For example $\mathrm{HCoV}-229 \mathrm{E}$ or SARS-CoV combined with a soluble C-type lectin might be able to enter cells that do not express a specific viral receptor such as hAPN or ACE2.

Table 2. Soluble CD209L binding to cell lines.

\begin{tabular}{lcc}
\hline Cell line & $\begin{array}{c}\text { [sCD209L] } \\
\mu \mathrm{g} / \mathrm{ml}\end{array}$ & $\begin{array}{c}\text { \% Cells that bind } \\
\text { antibody to } \\
\text { CD209L* }\end{array}$ \\
\hline CHO & 0 & $<5$ \\
CHO & 200 & 93 \\
MRC5 & 0 & 7 \\
MRC5 & 200 & 94 \\
CHO/CD209L & 0 & 97 \\
CHO/CD209L & 200 & 99 \\
* Detected by flow cytometry with goat polyclonal antibody to the C \\
terminal domain of CD209L.
\end{tabular}


Table 3. Effects of soluble CD209L on binding of HCoV-229E virions to cell lines.

\begin{tabular}{lcc}
\hline Cell line & $\begin{array}{c}{[\mathrm{sCD} 209 \mathrm{~L}]} \\
\mu \mathrm{g} / \mathrm{ml}\end{array}$ & $\begin{array}{c}\text { \% Cells that bind } \\
\text { virus* }\end{array}$ \\
\hline CHO & 0 & 31 \\
$\mathrm{CHO}$ & 50 & $<5$ \\
$\mathrm{CHO}$ & 200 & 87 \\
MRC5 & 0 & 92 \\
MRC5 & 50 & 22 \\
MRC5 & 200 & 81 \\
CHO/CD209L & 0 & 94 \\
CHO/CD209L & 50 & 59 \\
CHO/CD209L & 200 & 96 \\
\hline * Virus binding was detected by flow cytometry with monoclonal \\
antibody 511H6 directed against the viral spike glycoprotein.
\end{tabular}

Surfactant protein D in the lungs a C-type lectin that contains a carbohydrate recognition domain similar to that of $\mathrm{CD} 209 \mathrm{~L}^{12}$ is an important innate host defense mechanism that binds to high-mannose glycans on the surfaces of bacteria and viruses. Bacteria and viruses coated by SP-D form aggregates and are phagocytosed by macrophages. Because soluble CD209L can bind to $\mathrm{HCoV}-229 \mathrm{E}$ virions and enhance binding of virions to receptor negative cells in culture, it is possible that SP-D could also bind to HCoV-229E and mediate the attachment and eventual entry of virus into macrophages and type II alveolar cells in the lung.

The National Center for Biotechnology Information database lists 47 sequences for CD209L (also called L-SIGN or DC-SIGNR) from humans, apes, and mice. Many mammalian species have homologues of C-type lectins such as CD209L, SP-D, and mannose binding lectin. These anchored or soluble $\mathrm{C}$-type lectins may act as a bridge to allow for the initial crossing of the species barrier by viruses. If the only block to infection is receptor usage, it is possible that an enveloped virus that normally infects a non-human species could use human CD209L as a weak receptor to enter a small number of human type II alveolar cells or macrophages. Virus mutants that could use a different receptor in the lung more efficiently might develop and these would have a selective advantage for growth in human lung. The selected virus mutants might then be able to spread from human to human using the new receptor.

Further study of CD209L interacting with HCoV-229E in mouse or hamster models will help to elucidate the significance of this receptor in coronavirus infection in vivo.

\section{ACKNOWLEDGMENTS}

We are grateful to Dr. T. Miura for insightful discussion and Dr. P. Talbot for the generous gift of the 511H6 hybridoma. This research was supported by NIH grant AI59576. S.A.J. was supported by NIH postdoctoral training grant 1 T32 AI07587. 


\section{REFERENCES}

1. W. Li, M. J. Moore, et al., Angiotensin-converting enzyme 2 is a functional receptor for the SARS coronavirus, Nature 426, 450-454 (2003).

2. S. K. Wong, W. Li, et al., A 193-amino acid fragment of the SARS coronavirus S protein efficiently binds angiotensin-converting enzyme 2, J. Biol. Chem. 279, 3197-3201 (2004).

3. D. Harmer, M. Gilbert, et al., Quantitative mRNA expression profiling of ACE 2, a novel homologue of angiotensin converting enzyme, FEBS Lett. 532, 107-110 (2002).

4. A. Marzi, T. Gramberg, et al., DC-SIGN and DC-SIGNR interact with the glycoprotein of Marburg virus and the S protein of severe acute respiratory syndrome coronavirus, J. Virol. 78, 12090-12095 (2004).

5. S. A. Jeffers, S. M. Tusell, et al., Cd2091 (L-Sign) is a receptor for severe acute respiratory syndrome coronavirus, Proc. Natl. Acad. Sci. USA 101, 15748-15753 (2004).

6. R. W. Doms and D. Trono, The plasma membrane as a combat zone in the HIV battlefield, Genes Dev. 14, 2677-2688 (2000).

7. M. T. Yu Kimata, M. Cella, et al., Capture and transfer of simian immunodeficiency virus by macaque dendritic cells is enhanced by DC-SIGN, J. Virol. 76, 11827-11836 (2002).

8. E. Lontok, E. Corse, et al., Intracellular targeting signals contribute to localization of coronavirus spike proteins near the virus assembly site, J. Virol. 78, 5913-5922 (2004).

9. A. G. Bost, E. Prentice, et al., Mouse hepatitis virus replicase protein complexes are translocated to sites of M protein accumulation in the ERGIC at late times of infection, Virology 285, 21-29 (2001).

10. C. L. Yeager, R. A. Ashmun, et al., Human aminopeptidase $\mathrm{N}$ is a receptor for human coronavirus $229 \mathrm{E}$, Nature 357, 420-422 (1992).

11. D. B. Tresnan, R. Levis, et al., Feline aminopeptidase N serves as a receptor for feline, canine, porcine, and human coronaviruses in serogroup I, J. Virol. 70, 8669-8674 (1996).

12. J. K. van de Wetering, L. M. van Golde, et al., Collectins: players of the innate immune system, Eur. $J$ Biochem. 271, 1229-1249 (2004). 